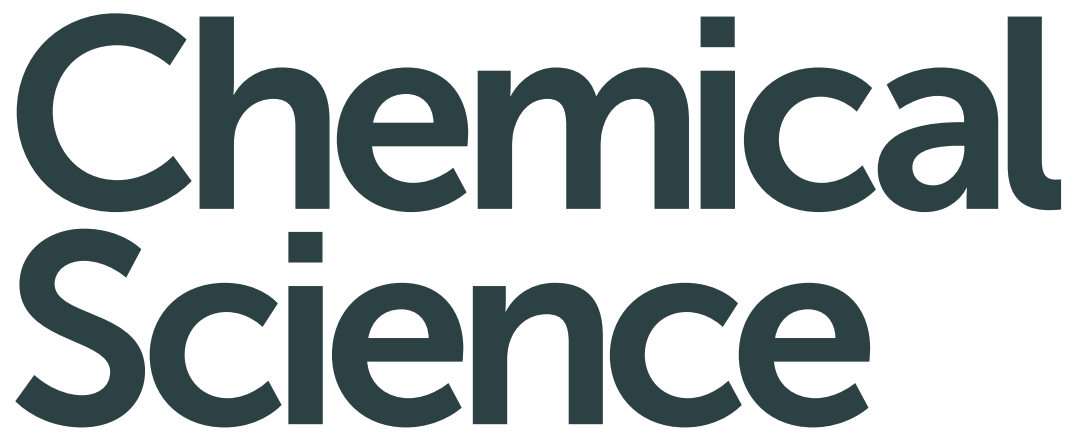

rsc.li/chemical-science
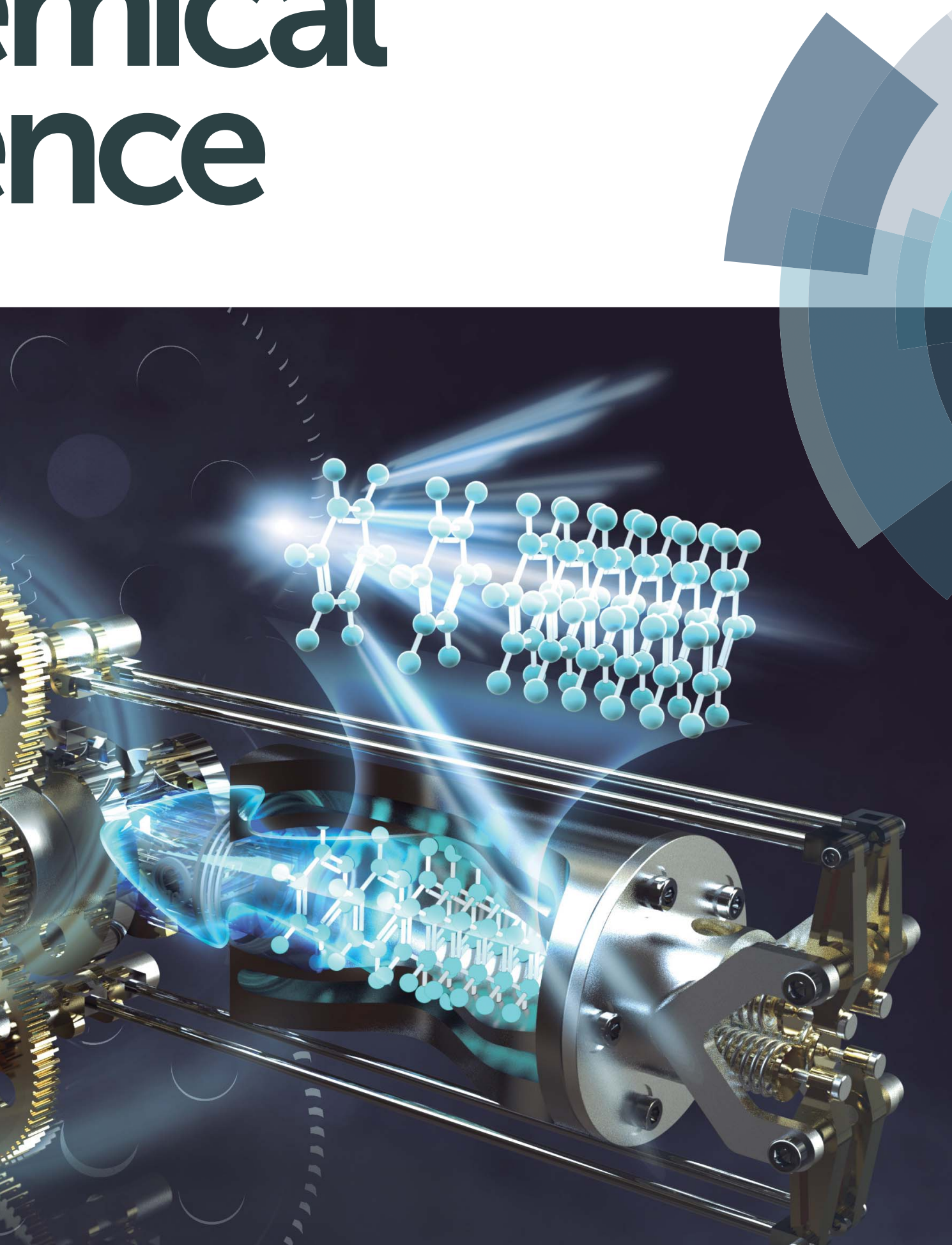
Check for updates

Cite this: Chem. Sci., 2019, 10, 7327

๑ All publication charges for this article have been paid for by the Royal Society of Chemistry

Received 20th May 2019

Accepted 29th June 2019

DOI: $10.1039 / c 9 s c 02444 a$

rsc.li/chemical-science

\title{
Martensitic organic crystals as soft actuators $\uparrow$
}

\author{
Liang Li, (D) $t^{\mathrm{a}}$ Patrick Commins, (D) $\ddagger^{\mathrm{a}}$ Marieh B. Al-Handawi, (D) ${ }^{a}$ \\ Durga Prasad Karothu, (D) a Jad Mahmoud Halabi, (D) a Stefan Schramm, (D) ${ }^{a}$ \\ James Weston, ${ }^{a}$ Rachid Rezgui (iD) ${ }^{a}$ and Panče Naumov (D) *ab
}

Being capable of rapid and complete structure switching, the martensitic phase transitions in molecular crystals are thought to hold a tremendous potential as thermally driven organic actuators. However, the mechanical engineering parlance in the assessment of their performance is not immediately legible to the chemistry research community that starts to explore these materials, and the unavailability of performance indices has precluded molecular crystals from being considered in the device design process. Here, we demonstrate that an organic martensite, hexamethylbenzene, can be used to perform work that is comparable to that of most actuator classes. Millimeter-size single crystals of this material undergo a transition between two forms by uniaxial expansion at a rate of $6.36(2) \mathrm{mm} \mathrm{s}^{-1}$, exerting force in the range $10-100 \mathrm{mN}$. The force-to-weight ratio of the crystals is on the order of $10^{4}$ and is superior to that of some living creatures. An actuator performance chart reveals that the performance of this material is close to that of nanomuscles, electrostatic actuators and voice coils, with a strain higher than that of electro/magnetostrictive actuators and ceramic piezoelectrics and stress higher than that of the electroactive polymers, MEMS devices, nanomuscles, voice coils, and some solenoids. Moreover, the crystals of this material are mechanically compliant and can be reversibly bent and shaped to fit the desired application. Altogether, the results point to the untapped potential of molecular crystals as rapid and efficient soft, organic actuators.

\section{Introduction}

The materials class of actuators embodies simple materials, multicomponent mechanical devices and complex biological systems that act as energy transducers and effectively convert energy from an external source into mechanical energy in a controlled fashion. ${ }^{1-13}$ Artificial actuators can be integrated as active elements in mechanically coupled mechatronic or adaptronic systems to convert electrical, thermal or light energy into work. The input varies according to the internal conversion process-electromagnetic, piezoelectric and magnetostrictive actuators use current, charge or voltage, the pneumatics use fluid pressure or flow, and shape memory and thermal expansion actuators use heat. Input parameters are provided by an external control system which optimizes the output of the actuator, while the force, work and stroke are recorded as output parameters that are essential for optimization of the

\footnotetext{
${ }^{a}$ New York University Abu Dhabi, Abu Dhabi, POB 129188, United Arab Emirates. E-mail: pance.naumov@nyu.edu

${ }^{b}$ Radcliffe Institute for Advanced Study, Harvard University, 10 Garden St., Cambridge, MA 02138, USA

$\dagger$ Electronic supplementary information (ESI) available: Experimental details and characterization data. CCDC 1936559. For ESI and crystallographic data in CIF or other electronic format see DOI: 10.1039/c9sc02444a

\$ These authors contribute equally to this work.
}

actuator's performance. The bimetallic ${ }^{14}$ and shape-memory ${ }^{15}$ actuators are rather inefficient because they require substantial energy due to heating or cooling. The piezo ${ }^{16}$ and magnetostrictive ${ }^{16,17}$ actuators have high power outputs and can operate at high frequencies, however they can only exert small strokes. The recent realization that mechanical effects observed with small-molecule adaptive molecular crystals could be a new platform for designing all-organic micro- or nanoactuating systems has also raised questions regarding the appropriateness of these hitherto unutilized materials for integration in soft devices beyond the simple demonstrations of the ability of such crystals to move or lift objects.

Here, we demonstrate that a crystal of a simple organic compound, hexamethylbenzene (HMB), can be used as allorganic actuator with a performance that is superior to many of the existing simple and complex actuating systems, and we set performance indices that are relevant to benchmark dynamic molecular crystals against other existing actuator classes. The mechanical properties of molecular crystals are directly related to the strength and directionality of their intermolecular interactions, which are generally weak compared to the covalent bonds. Unraveling the sole potential of the weak intermolecular interactions in organic crystals as actuators requires a material which does not include other, stronger intermolecular bonds. Being devoid of functional groups capable of hydrogen bonding, the crystal packing of 
HMB is governed entirely by weak interactions $(\pi-\pi$ stacking and van der Waals interactions), and thus it appeared ideal to explore the true potential of weak interactions. This material could provide insight into the lower performance limits of allorganic crystal as actuators.

\section{Results}

HMB has had a remarkable history. Polymorph II of HMB marks a milestone in the history of crystallography and organic chemistry as its structure was used in Lonsdale's seminal work as direct evidence of the planarity and aromaticity of the benzene ring. ${ }^{18}$ Two other polymorphs, a high-temperature phase (form I) ${ }^{19}$ and a low-temperature phase (form III) ${ }^{20}$ were discovered after more advanced crystallographic techniques became available. Single crystals of HMB used in this work were of form II, and their phase identity was confirmed by single crystal X-ray diffraction analysis (for details, see ESI, Table S1†). They were grown by slow evaporation of a solution of freshly sublimed sample in benzene layered on a water surface at room temperature (Note $\mathrm{S} 1 \dagger){ }^{21}$ This method provided unobstructed growth and prevented development of crystal defects in contact with the container surface, and afforded high-quality large single crystals. The crystals were transparent colorless parallelepipeds of several millimeters in length (Fig. 1A). Their thermal behavior at constant temperature and upon heating is shown in Fig. S4 and S5. $\dagger$

Form II is converted to form I by a first-order martensitic phase transition (Fig. 1A and B), ${ }^{22}$ which was noted for its unconventional properties and has been a subject to different mechanistic interpretations. ${ }^{19}$ As it was confirmed by DSC (Fig. $\mathrm{S} 1 \dagger$ ), form II transitions to form I at $382-385 \mathrm{~K}$, and the reverse phase transition, from form I to II, occurs at 380-382 K. In form II, the HMB molecules are assembled in layers that are parallel to the (111) plane and the molecular layers are stacked along the [100] direction (Fig. 1E and F). The transition to form I is accompanied by a small structural change; the distance between the molecular layers parallel to (111) increases from $3.67(1)$ to $3.76(1) \AA$, while the benzene rings practically retain their orientation relative to each other, with the difference in orientation between the rings in the two structures being only $1.04(1)^{\circ}$. For a $3.32(1) \mathrm{mm} \times 1.83(1) \mathrm{mm}([100] \times[110])$ crystal, this expansion of $5 \%$ results in elongation of the crystal along the $a$ axis ([100]) from 3.32(1) $\mathrm{mm}$ to $3.50(1) \mathrm{mm}$. The expansion of the crystals along the crystallographic $a$ axis during the transition appealed to us as potentially useful for inducing motion of other objects, that is, actuation.

The phase transition of a crystal of form II that was heated up to $393 \mathrm{~K}$ was recorded by using a hot-stage microscope coupled with a high-speed camera (Movie $\mathrm{S} 1 \dagger$ ). The new phase evolves at one end of the crystal, and the moving phase front is parallel to the (111) layers. The front propagates along the [100] direction (corresponding to the $a$ axis) at a rate of $6.36(2) \mathrm{mm}$ $\mathrm{s}^{-1}$. This speed of progression is slightly faster than that of other martensitic organic crystals, for instance the phase transition in di-tert-butyl[1] benzothieno[3,2-b][1]1-benzothiophene, which propagates at a rate of $0.807 \mathrm{~mm} \mathrm{~s}^{-1} \cdot{ }^{23}$ However, the rate of transition of HMB is two orders of magnitudes slower compared to that of thermosalient transitions, such as those of (phenylazophenyl)palladium hexafluoroacetylacetonate and Lpyroglutamic acid crystals that transition at rates of 540 and $427 \mathrm{~mm} \mathrm{~s}^{-1}$, respectively. ${ }^{24,25}$ This difference in the phase rate with thermosalient solids is consistent with the observation that although the transition in HMB is fast and martensitic, it is not thermosalient and does not result in self-propulsion of the crystal.

The elastic energy accumulated during the phase transition due to the expansion along the [100] direction is released as mechanical energy that can be used to perform work, and therefore we sought to determine the force generated during crystal expansion. The crystal was placed onto a tensile tester, sandwiched in between the instrument and a jig, and the experimental setup was heated. As the steel surface of the tester was heated, the thermal expansion of the steel was observed as gradual increase in load (Fig. 1D). At approximately $383 \mathrm{~K}$, the crystals transitioned from phase II to phase I and expanded, resulting in increase of the load. Three crystals of size $2.45 \times$ $1.49 \times 0.27 \mathrm{~mm}, 1.90 \times 0.66 \times 0.46 \mathrm{~mm}$ and $1.44 \times 1.38 \times$ $0.39 \mathrm{~mm}$ exerted forces of $41.5,42.4$ and $132.1 \mathrm{mN}$, respectively (Fig. 1D, S2 and S3, and Movies S2-S4 $\dagger$ ). After the transition, a consistent drop in load was detected, caused by gradual sublimation of the crystal from its corners (Fig. S4 and S5†) and the unbalanced contact of the subliming crystal with the tensile tester.

One of the key setbacks in considering organic crystals as actuators is that the physical properties and performance of single crystals are rarely reported in reference to their multicomponent competitors (actuating devices). The performance indices of an actuator are combinations of characteristics that reflect on the actuator's effectiveness in performing certain functions. Given an application, these indices play an important role in guiding the selection of the appropriate actuator. In comparing different systems based on performance indices, those which lie within an order of magnitude of each other are considered competitors. Maximum actuation stress $\sigma_{\max }$ and maximum actuation strain $\varepsilon_{\text {max }}$ are basic characteristics that limit the force and displacement, and consequently the maximum work output, produced by an actuator in a single stroke. A stroke in this case is defined as the uniaxial expansion induced by phase transition in the molecular crystal upon exposure to heat until the system stabilizes.

$$
\varepsilon_{\max }=\frac{L_{\mathrm{f}}-L_{\mathrm{i}}}{L_{\mathrm{i}}}
$$

where $L_{\mathrm{i}}$ and $L_{\mathrm{f}}$ are respectively the initial and final lengths of the crystal measured along the actuation axis before and after the phase transition is induced.

$$
\sigma_{\max }=\frac{F_{\max }}{A}
$$

where $F_{\max }$ is the maximum force produced after the completion of the phase transition and $A$ is the cross-sectional area of contact between the actuator and the probe or an object subject to the force. 

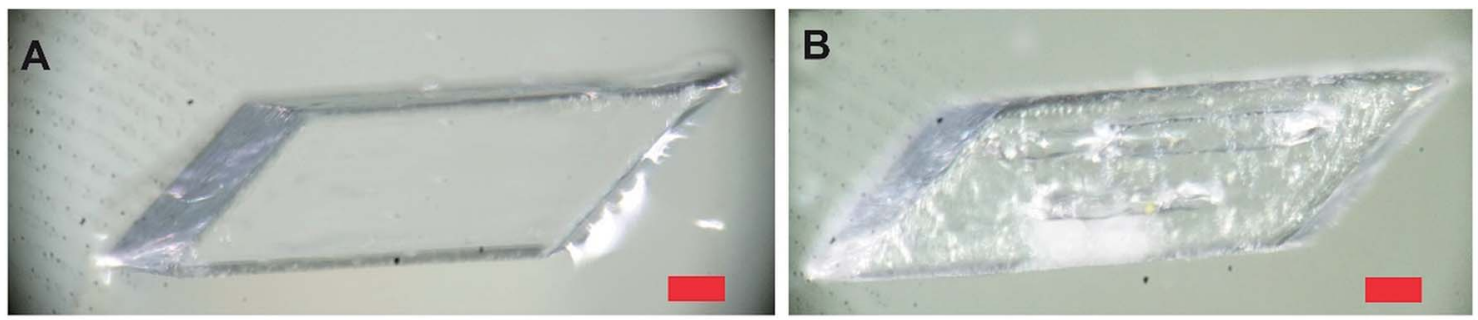

C

D
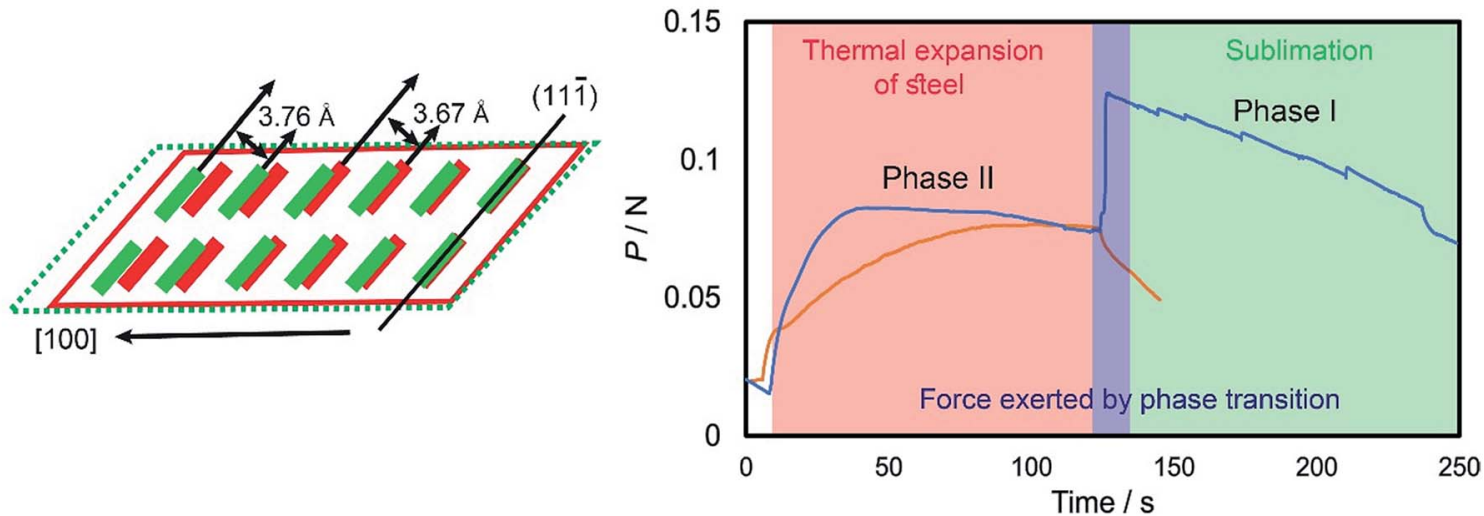

$\mathbf{E}$

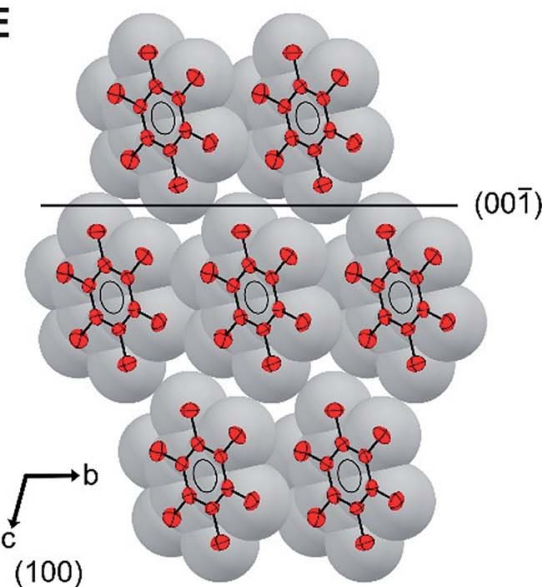

$\mathbf{F}$

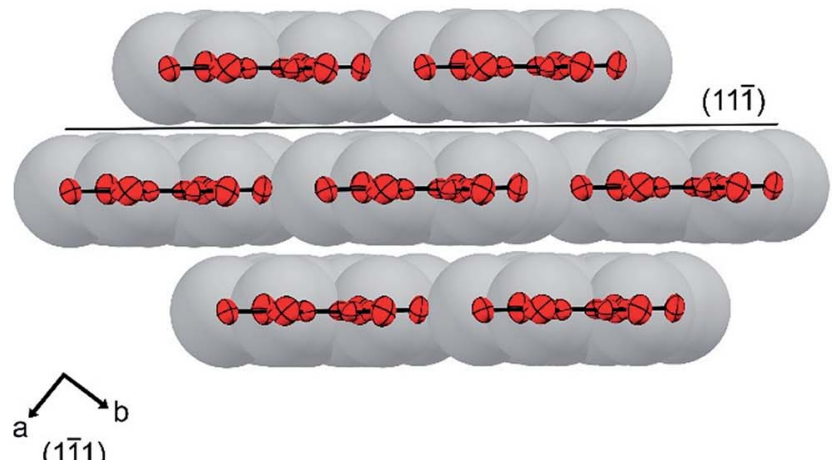

Fig. 1 Phase transition between forms II and I of hexamethylbenzene (HMB) and the related structural changes. (A and B) Optical image of $H M B$ crystal in form II (A) and form I (B) (scale bar: $500 \mu \mathrm{m}$ ). (C) Schematic representation of the overall crystal change upon transition between form II (red model) and form I (green model), which results in expansion on heating or contraction on cooling of the crystal in the [100] direction. The hydrogen atoms are omitted for clarity. (D) Load-time profile, monitored by using a heated tensile tester, recorded with (blue trace) and without (orange trace) a single crystal of HMB on the instrument. (E and F) Molecular packing of HMB molecules in the structure of form II viewed on (100) (E) and (111) (F). The atoms are shown as red thermal ellipsoids (red) with $50 \%$ probability encased by the respective van der Waals radii (grey). The gliding plane (111) is highlighted in panel $f$. The hydrogen atoms, which are omitted for clarity, are located in the space between the coplanar rings.

Fig. 2 compares the actuator stress and strain of HMB crystals to the other standard actuator classes. ${ }^{26}$ We infer that, despite being a homogeneous material of uniform composition, HMB performs similarly to other thermal actuators and it occupies a materials property space close to nanomuscles, electrostatic actuators and voice coils. A single crystal of HMB has properties similar to other thermal actuators that are based on metals or alloys. In regard to the maximum strain, HMB performs better than electrostrictive and magnetostrictive actuators, ceramic piezoelectrics, QuickPack actuators, disk translators, and even some MEMS devices. HMB exerts higher stress than electroactive polymers, MEMS devices, nanomuscles, voice coils, and even some solenoids. Within a more general context, the plot in Fig. 2 shows that single crystals have comparable actuating properties to other commonly known actuators. We contend that their underutilization in devices is not related to their properties per se, but rather due to the fact that comparative analysis of these properties has not been 


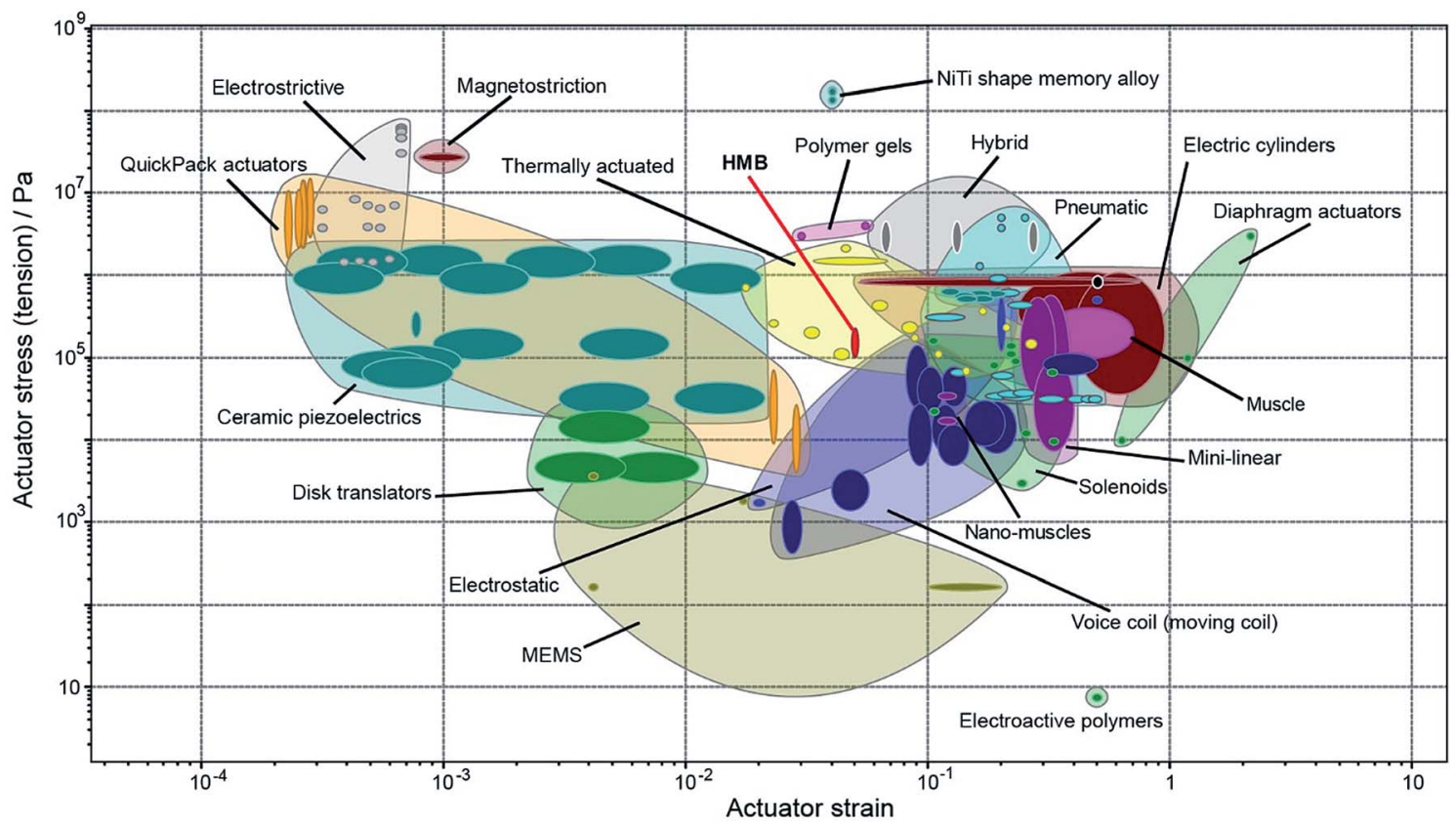

Fig. 2 Comparison of the maximum stress-strain profile of HMB and the common actuator classes. The strain is dimensionless. The envelopes for each actuator class, including HMB, encircle the maximum and minimum values of the strain and stress for each reported class but do not reflect the statistical spread of values or standard deviations within each class.

conveniently performed, displayed, and interpreted. Other important qualities such as reversibility, cyclability (fatigue), accumulation of defects, response time, efficiency in energy conversion, and other performance indicators must be met before an organic material is considered as actuator for real applications. Even that HMB is lacking some of these properties due to its softness that could be challenging for device fabrication and sublimation that limits the number of cycles, other organic crystals may not have these limitations and could have more suitable mechanical properties.

Comparison of the actuating ability of HMB single crystals expressed as force-to-weight ratio with some common biological systems such as animals further demonstrates their impressive performance (Fig. 3A and Table S2 $\dagger$ ). ${ }^{27-31}$ For example, ants are commonly considered strong as they are able to lift weights that are over 1000 times their own weight. Crystals of HMB are capable to exert a significant force relative to their weight. Three crystals of weights $0.40 \mathrm{mg}(3.9 \mu \mathrm{N}), 0.84 \mathrm{mg}(8.2 \mu \mathrm{N})$ and $1.05 \mathrm{mg}(10.2 \mu \mathrm{N})$ were able to exerts forces of $42.4,41.5$, and $132.1 \mathrm{mN}$, respectively, which equals an average force-to-weight ratio of $10212: 1$. This translates into an HMB crystal being able to apply more than 10000 times force its own weight. In order to visually demonstrate this actuation capability of HMB, we have designed a setup where a single crystal of HMB works as actuator (Fig. 3B-F and Movie S5 $\dagger$ ). A stainless steel ball, approximately $3 \mathrm{~mm}$ in diameter and $110 \mathrm{mg}$ in weight was placed on a flat surface close to one of the sharp tips of a single crystal of HMB and on a platform above set of parallel, dominolike glass blocks. Upon heating, the HMB crystal expands, pushing the ball which rolls and falls off the platform. This action triggers a domino-like effect with the blocks. This simple
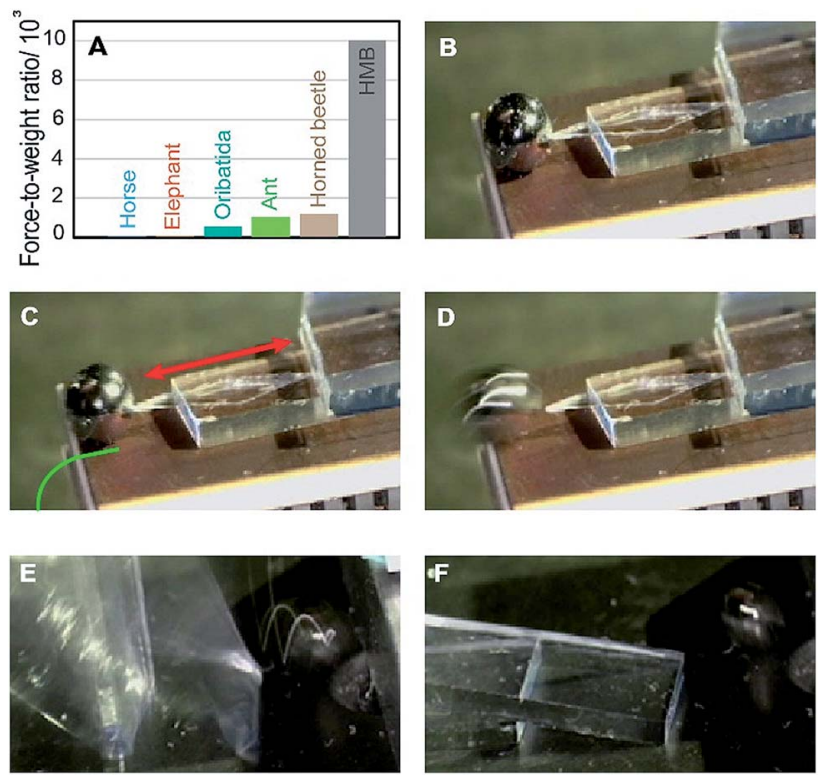

Fig. 3 Comparison of the performance of HMB single crystal with some biological systems and demonstration of its actuating ability. (A) Force-to-weight ratio of $\mathrm{HMB}$ and some living creatures. (B-F) Experimental setup with $\mathrm{HMB}$ single crystal acting as an actuator. The process starts with a steady setup which is heated (B), whereby the crystal of HMB expands and pushes a stainless steel ball (C). The ball rolls (D), falls of the platform (E) and triggers collapse of a series of glass blocks in a domino-like manner (F). The red arrow in panel (C) shows the direction of expansion of the crystal, and the green line shows the path of the ball motion. 


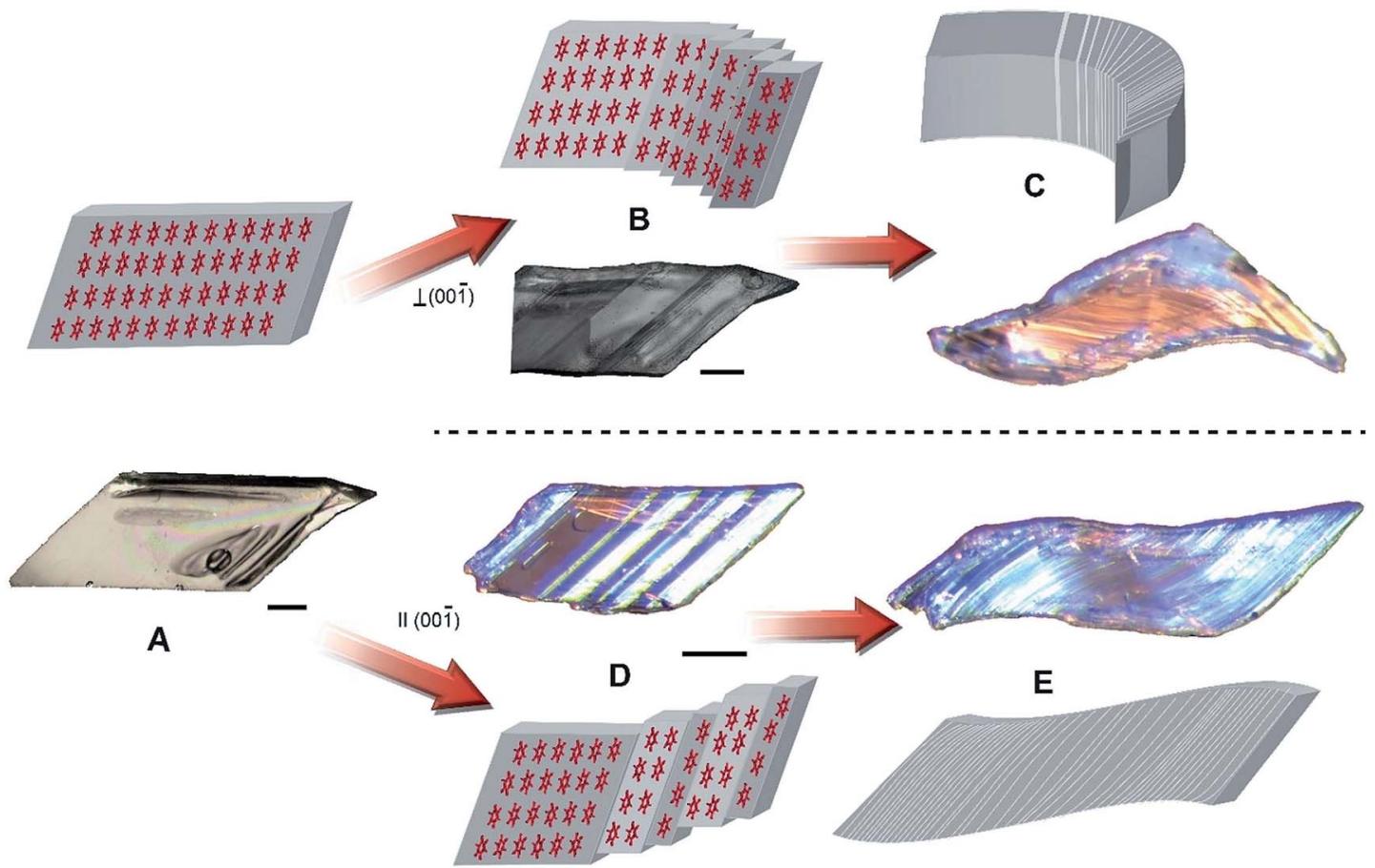

Fig. 4 Mechanical compliance of HMB crystals. (A) Optical image of a typical crystal, and molecular packing. HMB molecules are shown as sticks model (the bond radius is plotted as $0.2 \AA$ ) and the hydrogen atoms are omitted for clarity. (B-E) Optical images and cartoons of the evolution of twinned domains that evolve after force has been applied to the crystal normal (B and C) or parallel (D and E) to the (001) plane. The length of the scale bars in all panels is $100 \mu \mathrm{m}$.

demonstration illustrates potential future application of this and possibly other similar organic martensitic materials for actuation of other static objects that are much bigger in size than their own.

Noteworthy is the apparent non-linear variation of the force across the crystals of varying weight for the three samples. The force exerted by an expanding crystal depends, inter alia, on the polymorph, size of the crystal, contact surface area, and crystal face on which it is measured. It was shown recently that, provided all other conditions are retained identical, for crystals of different sizes, there is generally a fairly linear relationship between the force exerted on a certain crystal face and the crystal weight (see Fig. 3 in ref. 32). Although similar correlation can be expected in case of HMB, the small number of samples and the difficulties to attain control over the crystal, in addition to the always present defects, prevented us from establishing similar correlations in this case. The experimental uncertainty in the force measurement was generally low, and was within $0.5 \%$ of the measured values.

In addition to their actuation abilities, the crystals of $\mathrm{HMB}$ are also endowed with extraordinary mechanical compliance. They are soft to touch and bend readily when subject to local force, whereupon they delaminate into multiple twinned slabs (Movies S6 and S7, Note S2 $\dagger$ ). The mechanism of crystal bending is visualized in Fig. 4. The molecular layers interact weakly between the molecules in the (111) plane, and when force is applied either parallel or perpendicular to the $(00 \overline{1})$ plane the molecules glide over each other and generate twin domains. Bending parallel or perpendicular to the (001) plane is caused by formation of new twin domains and migration of shear dislocations that eventually cause delamination in the (111) plane, which appears as sliding of the adjacent slabs (Fig. 4B and D). When additional force is applied to the crystal, numerous twinned domains are formed in response by sliding of the molecules along the (111) plane and allow the crystal to bend or to be shaped in an S-like object (Fig. 4C and E). The formation of twin domains is reversible, and the original parent domain can be partially recovered simply by applying force in the opposite direction (Movies S8 and S9†).

\section{Conclusions}

In summary, the results presented herein show that crystals of a simple organic compound that undergoes a martensitic transition such as HMB can be used as all-organic actuators. Although the transition in this compound is slower than those in the thermosalient materials, it can provide stress and strain that are comparable to those of some common actuators classes and are similar to nanomuscles, electrostatic actuators and voice coils. During the transition, the crystal exerts mechanical force that is greater than 10000 times its own weight, and therefore exceeds many of the known living systems. These results indicate that, being endowed with long-range ordered structure, organic crystals hold an unexplored potential as soft actuators. Further studies are needed to assess their true potential in milli/ microscale mechatronic and adaptronic systems. 


\section{Conflicts of interest}

The authors declare no conflict of interest.

\section{Acknowledgements}

The work was carried out at New York University Abu Dhabi, using the Core Technology Platform (CTP) resources. The continuous support of Dr Reza Rowshan during the course of this project is greatly appreciated.

\section{Notes and references}

1 D. Stock, A. G. Leslie and J. E. Walker, Science, 1999, 286, 1700.

2 X. Dong, F. Tong, K. M. Hanson, R. O. Al-Kaysi, D. Kitagawa, S. Kobatake and C. J. Bardeen, Chem. Mater., 2019, 31, 1016. 3 R. O. Al-Kaysi, A. M. Müller and C. J. Bardeen, J. Am. Chem. Soc., 2006, 128, 15938.

4 S. C. Sahoo, N. K. Nath, L. Zhang, M. H. Semreen, T. H. Al-Tel and P. Naumov, RSC Adv., 2014, 4, 7640.

5 T. Minamino, K. Imada and K. Namba, Curr. Opin. Struct. Biol., 2008, 18, 693.

6 H. Noji, R. Yasuda, M. Yoshida and K. Kinosita, Nature, 1997, 386, 299.

7 T. Atsumi, L. McCartert and Y. Imae, Nature, 1992, 355, 182.

8 L. Zhang, H. Liang, J. Jacob and P. Naumov, Nat. Commun., 2015, 6, 7429.

9 M. A. C. Stuart, W. T. S. Huck, J. Genzer, M. Müller, C. Ober, M. Stamm, G. B. Sukhorukov, I. Szleifer, V. V. Tsukruk, M. Urban, F. Winnik, S. Zauscher, I. Luzinov and S. Minko, Nat. Mater., 2010, 9, 101.

10 Z. Liu and P. Calvert, Adv. Mater., 2000, 12, 288.

11 L. V. Schäfer, E. M. Müller, H. E. Gaub and H. Grubmüller, Angew. Chem., Int. Ed., 2007, 46, 2232.
12 O. Kim, T. J. Shin and M. J. Park, Nat. Commun., 2013, 4, 2208.

13 T. Mirfakhrai, J. D. W. Madden and R. H. Baughman, Mater. Today, 2007, 10, 30.

14 K. Horikawa, K. Yamaue and H. Kobayashi, Mater. Trans., 2010, 51, 2181.

15 K. Otsuka and C. M. Wayman, Shape memory materials, Cambridge University Press, Cambridge, 1998.

16 S. R. Anton and H. A. Sodano, Smart Mater. Struct., 2007, 16, R1.

17 E. Quandt and A. Ludwig, Sens. Actuators, A, 2000, 81, 275.

18 K. Lonsdale, Nature, 1928, 122, 810.

19 Y. Saito, $X$-RAYS, 1952, 7, 9.

20 J. Stride, Acta Crystallogr., Sect. B: Struct. Sci., 2005, 61, 200.

21 K. Izumi, J. Cryst. Growth, 1996, 169, 325.

22 Y. V. Mnyukh, N. A. Panfilova, N. N. Petropavlov and N. S. Uchvatova, J. Phys. Chem. Solids, 1975, 36, 127.

23 H. Chung, C. Ruzié, Y. Geerts and Y. Diao, Cryst. Growth Des., 2018, 18, 4245.

24 M. K. Panda, T. Runčevski, S. C. Sahoo, A. A. Belik, N. K. Nath, R. E. Dinnebier and P. Naumov, Nat. Commun., 2014, 5, 4811.

25 M. K. Panda, T. Runčevski, A. Husain, R. E. Dinnebier and P. Naumov, J. Am. Chem. Soc., 2015, 137, 1895.

26 J. E. Huber, N. A. Fleck and M. F. Ashby, Proc. R. Soc. London, Ser. A, 1997, 453, 2185.

27 R. D. Stevenson and R. J. Wassersug, Nature, 1993, 364, 195.

28 J. Shoshani, Trends Ecol. Evol., 1998, 13, 480.

29 M. Heethoff and L. Koerner, J. Exp. Biol., 2007, 210, 3036.

30 J. Wojtusiak, E. J. Godzińska and A. Dejean, Trop. Zool., 1995, 8, 309.

31 R. J. Knell and L. W. Simmons, Proc. R. Soc. B, 2010, 277, 2347.

32 A. Khalil, D. P. Karothu and P. Naumov, J. Am. Chem. Soc., 2019, 141, 3371. 